Address for Correspondence: Dr. Shahinul Alam, MBBS, MD, FCPS, Associate Professor, Department of Hepatology, Bangabandhu Sheikh Mujib Medical University, Shahbagh, Dhaka-1000, Bangladesh.

Email: Shahinul67@yahoo.com

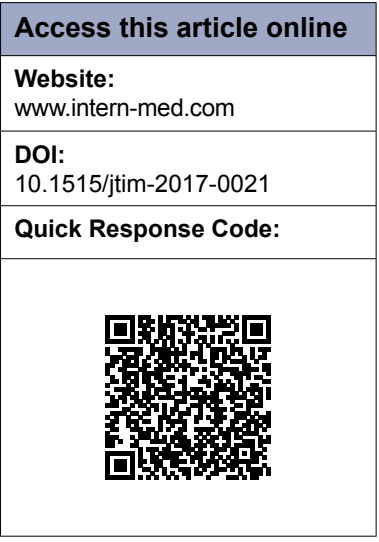

\title{
Effect of pentoxifylline on histological activity and fibrosis of nonalcoholic steatohepatitis patients: A one year randomized control trial
}

\author{
Shahinul Alam, SKM Nazmul Hasan, Golam Mustafa, Mahabubul Alam, \\ Mohammad Kamal, Nooruddin Ahmad \\ Department of Hepatology, Bangabandhu Sheikh Mujib Medical University, Shahbagh, Dhaka-1000, Bangladesh
}

\section{ABSTRACT}

Background and Objectives: To observe the effect of Pentoxifylline for 1 year on hepatic histological activity and fibrosis of nonalcoholic steatohepatitis (NASH). Materials and Methods: A single center, open label Randomized Control Trial. Patients were included if they had ultrasonographic evidence of fatty liver and nonalcoholic fatty liver disease activity score (NAS) $\geq 5$ on liver histology. A total of 35 patients were selected; 25 of PL (Experimental) group and 10 of $L$ (Control) group. PL group received 400 mg pentoxifylline thrice daily along with lifestyle modification and there was only lifestyle modification for the $L$ group. After one year, NAS and fibrosis was compared in both groups. Results: In PL group, NAS improved $2.10 \pm 1.07$; whereas in $\mathrm{L}$ group, NAS was $0.90 \pm 0.99(P=0.006)$. As per the protocol analysis, NAS $\geq 2$ improved in $15 / 20(75 \%)$ in PL group and in $3 / 10(30 \%)$ in $L$ group $(P=0.018)$. In PL group, the individual component of NAS, steatosis improved from $2.30 \pm 0.66$ to $0.95 \pm 0.76(P=0.000)$, lobular inflammation from $1.65 \pm 0.59$ to $1.05 \pm 0.51(P=0.002)$ and hepatocyte ballooning from $1.50 \pm 0.51$ to $1.30 \pm 0.57(P=0.258)$. In $L$ group, steatosis improved from $2.30 \pm 0.68$ to $1.40 \pm 1.08(P=0.01)$, lobular inflammation and hepatocyte ballooning did not improve. The fibrosis score did not improve in any group. In PL group, NAS improved significantly $(P=0.027$; $\mathrm{OR}=22.76, \mathrm{Cl}=1.43-362.40$ ) independent of weight reduction. Conclusion: Pentoxifylline for 1 year improves the hepatic histological activity but not fibrosis of NASH patients.

Key words: Pentoxifylline, NAFLD activity score, fibrosis score, NASH, fatty liver, Bangladesh

\section{INTRODUCTION}

Non-alcoholic fatty liver disease (NAFLD) is a condition pathologically linked to the metabolic syndrome by the intervention of insulin resistance (IR), characterized by hepatic steatosis in the absence of significant alcohol use, hepatotoxic medications or other known liver diseases. ${ }^{[1]}$ Globally, the prevalence of NAFLD is $25.24 \% 0^{[2]}$ In the Asia-Pacific region, the prevalence of NAFLD has increased remarkably over the years affecting up to $30 \%$ of the general population. ${ }^{[3]}$ In case of NAFLD, Bangladeshi ethnicity is an independent risk factor. ${ }^{[4]}$ The prevalence of NAFLD in general population of Bangladesh has been estimated to vary from 4 to $18.4 \%$, which jumps up to $49.8 \%$ in diabetic patients. ${ }^{[5,6]}$ Nonalcoholic steatohepatitis (NASH), the progressive form of NAFLD, is characterized by hepatocellular damage, inflammation and liver fibrosis that can progress to cirrhosis. ${ }^{[-9]}$ The pathogenesis of NASH is multifactorial, inflammatory activation clearly plays a pivotal role in the disease progression. Chronic inflammation interplaying with increased oxidative stress, cytokine production, direct lipotoxicity and autoimmunity is implicated in NAFLD pathophysiology by increasing NASH. Patients with NASH have significantly higher levels of serum TNF- $\alpha$ and IL-6 than seen in patients with simple steatosis. Cytokines including TNF- $\alpha$, a proinflammatory cytokine and adiponectin, an anti-inflammatory cytokine, are believed to play an important role in hepatocellular damage, inflammation and fibrogenesis in NASH. 
Currently, most hepatologists attempt to manage NASH by lifestyle changes such as weight reduction with or without exercise, as well as standard therapeutic interventions to control concomitant disease, for example, hyperlipidemia, hypertension and type 2 DM. Pentoxifylline (PTX) is known to decrease oxidative stress. ${ }^{[10]}$ PTX also has hydroxyl and peroxyl radical scavenging effects ${ }^{[11]}$ and specifically inhibits lipid peroxidation. ${ }^{[12]}$ Moreover, pentoxifylline has anti-inflammatory properties and it is also known to definitely suppress TNF- $\alpha$ gene transcription, preventing TNF $-\alpha$ synthesis. ${ }^{[13]}$ Therefore, PTX plays an important role in the inhibition of second hit hypothesis required for pathogenesis of NASH.

Only few studies and pilot trials of PTX ${ }^{[14-16]}$ suggested that pentoxifylline reduced plasma TNF- $\alpha$ and IL-6, ALT and AST levels but there is scarcity of data to observe improvement of NAFLD activity score (NAS) and fibrosis score in patients with nonalcoholic steatohepatitis (NASH). The aim of this study was to observe the effect of pentoxifylline on histological activity and fibrosis of Bangladeshi nonalcoholic steatohepatitis patients.

\section{MATERIALS AND METHODS}

This study was conducted from August 2014 to December 2015 as an open label Randomized Control Trial (RCT). The study was reviewed and approved by the Institutional Review Board (IRB)/Ethics committee of the university and was registered at Sri Lankan Clinical Trial Registry (SLCTR/ 2014/ 016). The aims and objectives of the study along with its procedure, risks and benefits of the study were explained to the study subjects and signed informed consent was taken from them, in accordance with the Helsinki declaration. The study subjects were assured about privacy, confidentiality, freedom to withdraw themselves at any time from the study and were also ensured that this would not be a barrier to get the available standard treatment.

Patient were deliberated for the study if they had ultrasonographic evidence of fatty liver and NAS greater than or equal to 5 on liver biopsy. Patients were excluded if there was any history of: 1) significant alcohol intake (more than $20 \mathrm{gm} /$ day); 2) taking drugs that may cause fatty liver (i.e., tamoxifen, valproic acid, amiodarone, methotrexate) or history of taking drugs that have shown benefit in previous NASH pilot studies (i.e., vitamin E, metformin, thiazolidinediones or fibrates); 3) chronic viral hepatitis (HBV or HCV); 4) pregnancy; 5) co-morbid condition (COPD, CKD, CCF or acute viral hepatitis.); 6) recent MI; 7) liver failure.

A total of 35 patients were selected for randomization; 25 of PL (Experimental) group and 10 of L (Control) group were followed for next one year. Five patients of PL group were lost from the study due to lack of interest of doing end of study liver biopsy. So, a total of 30 patients were considered for final analysis. PL group received $400 \mathrm{mg}$ pentoxifylline thrice daily along with lifestyle modification and there was only lifestyle modification for L group for one year. After one year, we repeated the liver biopsy in both PL and L group. All the patients of PL and L group were opened and at the same time researcher was also opened about PL and L group. Moderate exercise (30 minutes' walk a day) and dietary advice (avoidance of fatty foods as well as diet containing excessive sugar) was given in both groups. Diabetic patients were treated with lifestyle modification and if required, insulin secretagogue or insulin. Patients with dyslipidemia were initially treated by nonpharmacological measures for first three months. If any patient were still dyslipidemic (TC $>200 \mathrm{mg} / \mathrm{dl}$, TG > $150 \mathrm{mg} / \mathrm{dl}$ ), atorvastatin was added. Hypertensive patients were treated by antihypertensive drug except ACE inhibitor, ARB and calcium channel blocker (diltiazem). Close liaison was maintained with all patients. All patients were advised to contact immediately, if there was any problem.

\section{Biochemical analysis}

University Bio-chemistry laboratory was used for biochemical analysis. Fasting blood sugar (FBS), alanine aminotransferase (ALT), aspartate-aminotransferase (AST), gamma-glutamyl transpeptidase (GGT), bilirubin (B), total cholesterol (TC) and triglycerides (TG), LDL-C, HDL-C were determined on fresh serum using an autoanalyzer. Serum samples obtained after an overnight fast of at least $12 \mathrm{~h}$ and immediately frozen at -20 degree Celsius were used to determine the levels of immunoreactive insulin (IRI) by a chemiluminescent immunoassay. Homeostasis model assessment of Insulin resistance (HOMA-IR) was calculated by online HOMA 2 calculator. ${ }^{[17]}$

\section{Histopathology analysis}

All the liver biopsies were done as in-patient with full resuscitation facilities. The percutaneous liver biopsy technique was applied in all the cases. ${ }^{[18-20]}$ All biopsy specimens were fixed with $10 \%$ formalin and stained with hematoxylin-eosin and Masson's trichrome. The specimens were evaluated by experienced pathologists, not aware about the allocation of treatment and control group as well as about the clinical and biochemical parameters of any patient, using the scoring system validated by Kleiner et al. As known, this histology scoring system quantifies the necroinflammatory and steatotic changes (steatosis, lobular inflammation and ballooning) resulting in NAFLD activity scores (NAS) that ranged between 0 and 8 . Fibrotic changes were evaluated separately from NAS, ranging from 0 (no fibrosis) to 4 (cirrhosis). 


\section{Study schedule E surveillance parameters}

After screening, the included patients were followed for 12 months. The patients were followed monthly for initial 3 months, and then every 3 monthly for the next 9 months. Each visit took place between $10.00 \mathrm{am}$ to $02.00 \mathrm{pm}$ and consisted of a clinical examination, blood pressure (BP), body mass index (BMI) determinations and a questionnaire. Serum was collected for CBC with ESR, FBS, 2HABF, ALT, AST, PT with INR, GGT, bilirubin, total cholesterol, TG, HDL, LDL and IRI determinations in first and last visit. An alcohol consumption questionnaire was also administered and study compliance was strictly monitored. FBS, $2 \mathrm{HABF}$, lipid profile for diabetic and dyslipidemic patients were monitored as per the requirement. Additionally, the $1^{\text {st }}$ visit comprised of recording of the index liver biopsy, while last visit ended with the $2^{\text {nd }}$ liver biopsy, performed at maximum 2 weeks after the end-of treatment. The primary parameters that were compared between the first and last visit are SBP, DBP, WC, BMI, ALT, AST, GGT, HOMA-2 IR, TC, TG, HDL, LDL, FBS, 2HABF, NAS (including its components such as steatosis, ballooning and lobular inflammation) and fibrosis scores.

\section{Statistical analysis}

Quantitative data were presented as mean \pm SD and qualitative data were presented as percentage. All data were analyzed by SPSS version 20 (SPSS Inc.; Chicago, IL, US). Qualitative data analyzed by Chi-square test and quantitative data by Independent t-test, Man-Whitney U test and Paired t-test. All quantitative and qualitative data were analyzed between responders and non-responders. The univariate and multivariate logistic regression analysis was done to find out the best predictor of response. A statistically significant result was considered when $P$ value was less than 0.05 .

\section{RESULT}

\section{Patient base line characteristic, compliances, and follow-up}

A total of 30 patients (20 patients of PL group and 10 patients of $\mathrm{L}$ group) were considered for the final analysis (Figure 1). Five patients of PL group were lost from the study due to their lack of interest to adhere the study protocol. Twenty patients of PL group and 10 patients of L group completed the study according to the protocol and had done the $2^{\text {nd }}$ liver biopsy. There was no statistically significant difference regarding gender, serum bilirubin, ALT, AST, GGT, alkaline phosphatase, fasting lipid profile, fasting blood sugar, HOMA- IR, histological activity/ NAS and fibrosis score between the 2 groups of patients. But incidentally the patients of PL group were older, had higher $\mathrm{BMI}$ and higher waist circumference (Table 1).

\section{Histological response}

NAS improved in PL group from $5.45 \pm 0.76$ to $3.30 \pm$ $1.13(P=0.000)$ and in L group from $5.30 \pm 0.68$ to 4.20 \pm 1.23 ( $P=0.007)$. In PL group, the individual component of NAS, steatosis improved from $2.30 \pm 0.66$ to $0.95 \pm$ $0.76(P=0.000)$, lobular inflammation from $1.65 \pm 0.59$ to $1.05 \pm 0.51(P=0.002)$ and hepatocyte ballooning from $1.50 \pm 0.51$ to $1.30 \pm 0.57(P=0.258)$. In L group, steatosis improved from $2.30 \pm 0.68$ to $1.40 \pm 1.08(P=0.01)$, lobular inflammation from $1.50 \pm 0.53$ to $1.40 \pm 0.51(P=0.591)$ and hepatocyte ballooning from $1.50 \pm 0.52$ to $1.40 \pm 0.52$ $(P=0.678)$. NAS improvement in L group was due to the improvement of steatosis of 2 patients who had lost body weight of more than $7 \%$. Fibrosis score improved in PL group from $1.25 \pm 0.44$ to $1.20 \pm 0.70(P=0.716)$ and in L group it improved from $1.30 \pm 0.68$ to $1.30 \pm 0.48(P=$ 1.00). Improvement was not statistically significant (Table 2 ).

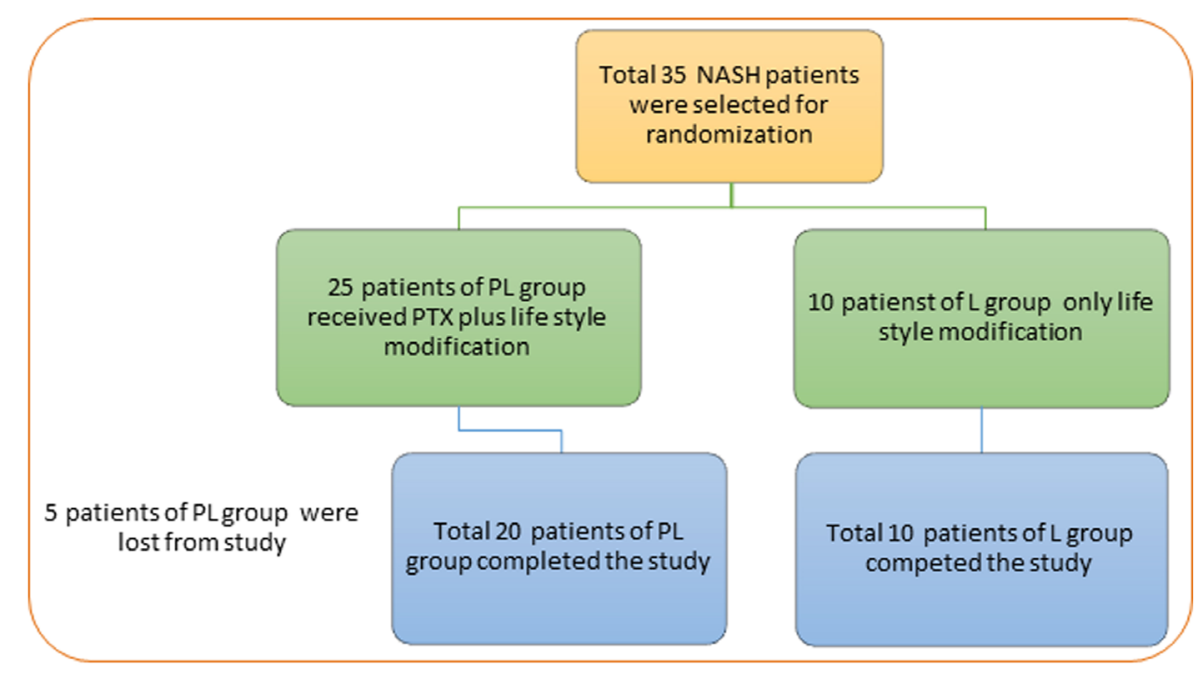

Figure 1: Consort flow chart of the study. 
Alam et al.: Pentoxifylline in Nonalcoholic Steatohepatitis

\begin{tabular}{|c|c|c|c|}
\hline Variables & $\begin{array}{l}\text { PL group } \\
(n=25) \\
\text { (mean } \pm \text { SD) }\end{array}$ & $\begin{array}{l}\text { L group } \\
(n=10) \\
(\text { mean } \pm \text { SD) }\end{array}$ & $P$-value \\
\hline Age (year) & $41.52 \pm 9.85$ & $38.80 \pm 6.18$ & 0.004 \\
\hline Sex (male/female) & $7 / 18(28 \% / 72 \%)$ & $5 / 5(50 \% / 50 \%)$ & 0.215 \\
\hline Diabetes (yes/no) & $8 / 17(32 \% / 68 \%)$ & $2 / 8(20 \% / 80 \%)$ & 0.478 \\
\hline Hypertension (yes/no) & $7 / 18(28 \% / 72 \%)$ & $3 / 7(30 \% / 70 \%)$ & 0.906 \\
\hline $\mathrm{BMI}\left(\mathrm{kg} / \mathrm{m}^{2}\right)$ & $27.97 \pm 3.33$ & $24.33 \pm 1.48$ & 0.002 \\
\hline WC $(\mathrm{cm})$ & $95.28 \pm 7.72$ & $89.40 \pm 3.80$ & 0.005 \\
\hline Bilirubin $(\mu \mathrm{mol} / \mathrm{L})$ & $9.88 \pm 3.24$ & $10.46 \pm 3.2$ & 0.640 \\
\hline $\mathrm{ALT}(\mathrm{U} / \mathrm{L})$ & $71.16 \pm 35$ & $57.10 \pm 30.12$ & 0.249 \\
\hline AST (U/L) & $43.96 \pm 28.26$ & $38.30 \pm 18.51$ & 0.493 \\
\hline GGT (U/L) & $63.24 \pm 43.19$ & $50.60 \pm 19.70$ & 0.244 \\
\hline $\operatorname{ALP}(\mathrm{U} / \mathrm{L})$ & $104.12 \pm 27.25$ & $83.12 \pm 39.97$ & 0.199 \\
\hline FBS $(\mathrm{mmol} / \mathrm{L})$ & $5.58 \pm 1.84$ & $5.37 \pm 1.94$ & 0.777 \\
\hline HOMA- IR & $2.37 \pm 1.33$ & $2.46 \pm 1.93$ & 0.907 \\
\hline Cholesterol (mg/dL) & $192.96 \pm 49.87$ & $204.5 \pm 48.04$ & 0.536 \\
\hline $\mathrm{LDL}(\mathrm{mg} / \mathrm{dL})$ & $108.86 \pm 43.58$ & $121 \pm 30.48$ & 0.393 \\
\hline $\mathrm{HDL}(\mathrm{mg} / \mathrm{dL}))$ & $37.38 \pm 9.13$ & $34.60 \pm 13.88$ & 0.571 \\
\hline Triglycerides (mg/dL) & $217.67 \pm 137.62$ & $299.30 \pm 261.57$ & 0.370 \\
\hline
\end{tabular}

WC: waist circumference; BMI: body mass index; ALT: alanine transaminase; AST: aspartate transaminase; ALP: alkaline phosphatase; GGT: gamma glutamyl transpeptidase; FBS: fasting blood sugar; HOMA-IR: homeostatic model assessment of insulin resistance; LDL: low density lipoprotein; HDL: high density lipoprotein.

NAS $\geq 2$ improved in 15 patients out of $20(75 \%)$ in PL group and in 3 patients out of $10(30 \%)$ in L group $(P=0.018)$ (Figure 2). Fibrosis score $\geq 1$ improved in 4 patients $(20 \%)$ in PL group, whereas in L group, 2 patients had this improvement (20\%). Fibrosis improved in total 6 patients; 4 in PL group and 2 in L group. The difference of fibrosis score $\geq 1$ improvement between two groups was not statistically significant $(P=1.00)$. In this study, NAS $\geq 2$ or fibrosis score $\geq 1$ improvement was considered as significant histological improvement (histological responder). Total 16 patients $(80 \%)$ were histological responder in PL group whereas there were $4(40 \%)$ in L group $(P=0.028)$

\section{Weight reduction and histological response}

BMI was improved from $27.43 \pm 3.03$ to $26.11 \pm 3.41(P$ $=0.007)$ and from $24.33 \pm 1.5$ to $23.89 \pm 1.90(P=0.306)$ in PL group and L group respectively (Table 3).

A total of 9 patients lost $\geq 7 \%$ bodyweight, among them $7(35 \%)$ in PL group and $2(20 \%)$ in L group. NAS $\geq$ 2 improvement was not associated with this amount of weight loss $(P=0.626)$ and fibrosis score $\geq 1$ improvement was not also associated with the above-mentioned weight loss $(P=0.426)$. NAS improved from $5.43 \pm 0.75$ to 3.67 $\pm 1.35(P=0.000)$ in $\geq 7 \%$ non-weight lost patient and it was from $5.33 \pm 0.71$ to $3.44 \pm 0.88(P=0.001)$ in $\geq 7 \%$ non-weight lost patients. With $\geq 7 \%$ weight loss, steatosis improved from $2.56 \pm 0.53$ to $1.22 \pm 0.97(P=$
0.000), but there was no significant improvement in lobular inflammation and ballooning. Without $\geq 7 \%$ weight lost patient, there was improvement of steatosis from $2.19 \pm$ 0.68 to $1.05 \pm 0.87(P=0.001)$ and lobular inflammation from $1.81 \pm 0.51$ to $1.29 \pm 0.56(P=0.008)$. Change of ballooning was not significant in these patients. Among patients with $\geq 7 \%$ body weight looser, $6(66.7 \%)$ were histological responders and $3(33.3 \%)$ were histological non-responders. On the other hand, those who did not lose $7 \%$ body weight, 14 (66.7\%) were histological responders and 7 (33.3\%) were histological non-responders (Figure 3). So, a significant body weight loss (7\% or more) was not associated with significant histological improvement $(P=$ $0.592)$.

\section{Biochemical Improvement}

ALT improved from $69.60 \pm 37.78$ to $36.20 \pm 20.26(P=$ $0.001)$ in PL group and $57.1 \pm 30.12$ to $32.6 \pm 13.08(P=$ $0.028)$ in L group, AST improved from $45.42 \pm 31.53$ to $24.26 \pm 10.26(P=0.008)$ in PL group and from $38.30 \pm$ 18.51 to $25.1 \pm 11.65(P=0.023)$ in L group and GGT from $61.67 \pm 48.60$ to $37.28 \pm 24.72(P=0.017)$ in PL group and from $50.60 \pm 19.74$ to $51.20 \pm 57.04(P=0.967)$ in $\mathrm{L}$ group and insulin resistance index improved from 2.53 \pm 1.50 to $2.12 \pm 1.31(P=0.432)$ in PL group and from $2.71 \pm 1.94$ to $1.61 \pm 0.59(P=0.233)$ in $L$ group. Serum cholesterol, Triglyceride and HDL did not improve in PL and L group. ALT, AST and GGT changes were not related to weight reduction. 
Alam et al: : Pentoxifylline in Nonalcoholic Steatohepatitis

\begin{tabular}{|c|c|c|c|c|c|c|}
\hline \multirow[t]{2}{*}{ Variables } & \multirow[b]{2}{*}{ Before Intervention } & \multicolumn{2}{|l|}{ PL group $(n=20)$} & \multicolumn{3}{|l|}{ L group $(n=10)$} \\
\hline & & After Intervention & $P$ value & Before intervention & After Intervention & $P$ value \\
\hline NAFLD activity score & $5.45 \pm 0.76$ & $3.30 \pm 1.13$ & 0.000 & $5.30 \pm 0.68$ & $4.20 \pm 1.23$ & 0.007 \\
\hline Steatosis & $2.30 \pm 0.66$ & $0.95 \pm 0.76$ & 0.000 & $2.30 \pm 0.68$ & $1.40 \pm 1.08$ & 0.01 \\
\hline Lobular inflammation & $1.65 \pm 0.59$ & $1.05 \pm 0.51$ & 0.002 & $1.50 \pm 0.53$ & $1.40 \pm 0.52$ & 0.591 \\
\hline Hepatocytes ballooning & $1.50 \pm 0.51$ & $1.30 \pm 0.57$ & 0.258 & $1.50 \pm 0.53$ & $1.40 \pm 0.52$ & 0.678 \\
\hline Fibrosis score & $1.25 \pm 0.44$ & $1.20 \pm 0.70$ & 0.716 & $1.30 \pm 0.68$ & $1.30 \pm 0.48$ & 1.00 \\
\hline ALT & $69.60 \pm 37.78$ & $36.20 \pm 20.26$ & 0.001 & $57.1 \pm 30.12$ & $32.6 \pm 13.08$ & 0.028 \\
\hline AST & $45.42 \pm 31.53$ & $24.26 \pm 10.26$ & 0.008 & $38.30 \pm 18.51$ & $25.1 \pm 11.65$ & 0.023 \\
\hline GGT & $61.67 \pm 48.60$ & $37.28 \pm 24.72$ & 0.017 & $50.60 \pm 19.74$ & $51.20 \pm 57.04$ & 0.967 \\
\hline
\end{tabular}

ALT: alanine transaminase; AST: aspartate transaminase; GGT: gamma glutamyl transpeptidase; NAFLD: non-alcoholic fatty liver disease.

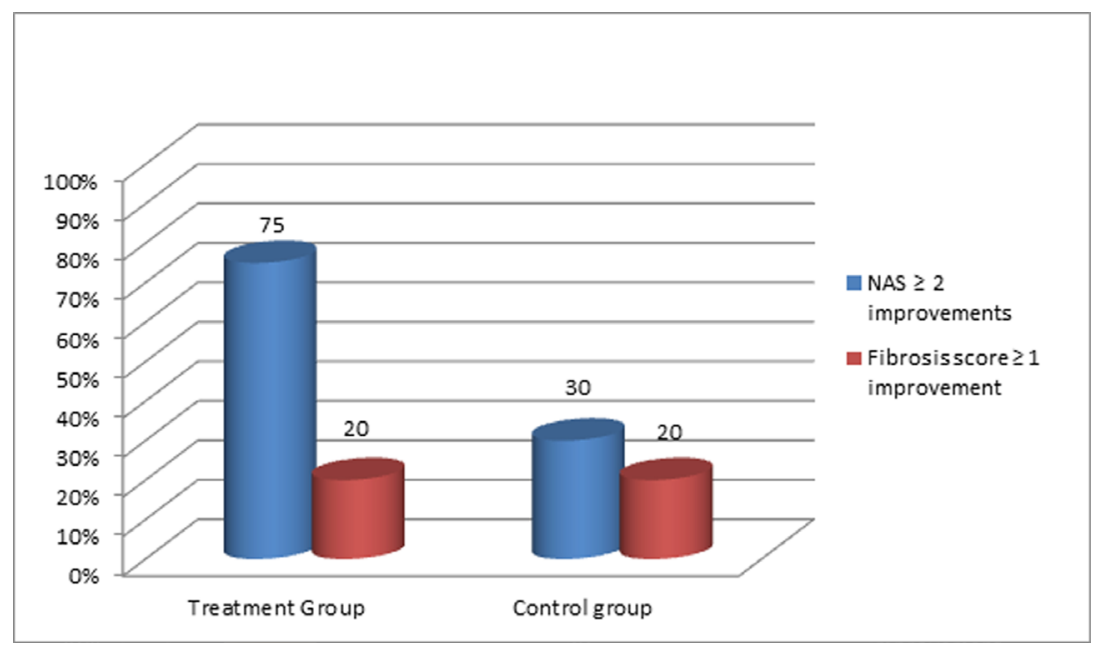

Figure 2: Distribution of NAS \& Fibrosis score improvement.

Comparison of histological, anthropometric and biochemical characteristic improvement from baseline

At the end of the study, NAS improved in both group; the difference of NAS improvement between two groups was statistically significant, $2.10 \pm 1.07$ versus $0.90 \pm 0.99$ ( $P$ $=0.006)$; significantly higher in PL group. But the fibrosis score improvement was $(0.05 \pm 0.60$ versus $0.00 \pm 0.57)$ and difference was not statistically significant $(P=0.829)$. There was no significant difference of improvement of BMI, WC $(P=0.205, P=0.151)$, ALT, GGT, FBS, TG and $\operatorname{SBP}(P=0.556, P=0.127, P=0.835, P=0.432)$ between PL and L group (Table III). HDL improvement between $\mathrm{PL}$ and $\mathrm{L}$ group was statistically significant $(P=0.043)$.

ALT was improved in both histological responder and nonresponder. GGT improved $24.06 \pm 38.14 \mathrm{U} / \mathrm{L}$ in responder and deteriorated $-0.45 \pm 48.74 \mathrm{IU}$ in non-responder, but not statistically significant $(P=0.148)$.

\section{Influence of pentoxifylline therapy on histological response}

Logistic regression analysis was done to find out the best predictor of patient response. All the important dynamic factors as well as the patient group were considered for logistic regression analysis. Univariate analysis explored that pentoxifylline had the effect on $(P=0.012$; OR $=9.33$, CI $=1.63-53.20)$ histological response. Other factors such as BMI improvement $(P=0.561)$, HOMA-2 IR improvement $(P=0.478)$ could not influence histological improvement (Table 4). Multivariate logistic regression analysis was done to see the effects of all confounding variable together. Multivariate analysis also explored that pentoxifylline significantly influenced the histological improvement $(P$ $=0.027$; OR $=22.76, \mathrm{CI}=1.43-362.40)$.

\section{Probable side effects and safety result}

Any adverse events were documented during one year of patient management. Most common side effects were abdominal pain and dyspepsia. In the PL group, 5 patients $(25 \%)$ developed abdominal pain, whereas in L group, 3 patients $(30 \%)$ developed abdominal pain $(P=0.086)$ (Table 5). On the other hand, 1 patient (5\%) in PL group and 2 patients $(20 \%)$ in L group had dyspepsia $(P=0.262)$. The occurrence of all possible side effects in PL and L group, could not reach statistically significant different level. No patient required treatment discontinuation due to drug related side effects. 
Alam et al.: Pentoxifylline in Nonalcoholic Steatohepatitis

\begin{tabular}{llll}
\hline \multicolumn{2}{l}{ Table 3: Mean change from baseline after 12 months } & & \\
\hline Improvement & PL group (mean \pm SD) & L group (mean \pm SD) & $P$ value \\
\hline NAS & $2.10 \pm 1.07$ & $0.90 \pm 0.99$ & 0.006 \\
Fibrosis score & $0.045 \pm 0.60$ & $0.00 \pm 0.57$ & 0.829 \\
BMI $\left(\mathrm{kg} / \mathrm{m}^{2}\right)$ & $1.33 \pm 1.96$ & $0.44 \pm 1.28$ & 0.205 \\
WC $(\mathrm{cm})$ & $2.95 \pm 3.67$ & $0.90 \pm 3.41$ & 0.151 \\
TG $(\mathrm{mg} / \mathrm{dL})$ & $33.44 \pm 157.50$ & $-28.22 \pm 186.23$ & 0.375 \\
Cholesterol $(\mathrm{mg} / \mathrm{dL})$ & $5.39 \pm 61.13$ & $-8.00 \pm 96.10$ & 0.662 \\
HDL $(\mathrm{mg} / \mathrm{dL})$ & $2.56 \pm 7.47$ & $-10 \pm 20.49$ & 0.043 \\
LDL $(\mathrm{mg} / \mathrm{dL})$ & $-2.67 \pm 59.42$ & $18.00 \pm 24.59$ & 0.361 \\
FBS $(\mathrm{mmol} / \mathrm{L})$ & $-0.09 \pm 0.80$ & $0.007 \pm 1.77$ & 0.835 \\
HOMA- IR & $0.418 \pm 2.03$ & $1.44 \pm 1.95$ & 0.273 \\
ALT (U/L) & $33.40 \pm 36.99$ & $25.46 \pm 29.04$ & 0.556 \\
GGT (U/L) & $23.83 \pm 37.08$ & $-2.5 \pm 50.79$ & 0.127 \\
SBP (mm Hg) & $3.50 \pm 18.07$ & $9.00 \pm 17.29$ & 0.432 \\
DBP (mm Hg) & $-3.00 \pm 7.14$ & $4.50 \pm 9.26$ & 0.021 \\
\hline
\end{tabular}

Abbreviation: NAS: NAFLD activity score; BMI: body mass index; WC: waist circumference; TG: triglycerides; HDL: high density lipoprotein; LDL: low density lipoprotein; FBS: fasting blood sugar; HOMA-IR: homeostatic model assessment of insulin resistance; ALT: alanine transaminase; GGT: gamma glutamyl transferase; SBP: systolic blood pressure; DBP: diastolic blood pressure.

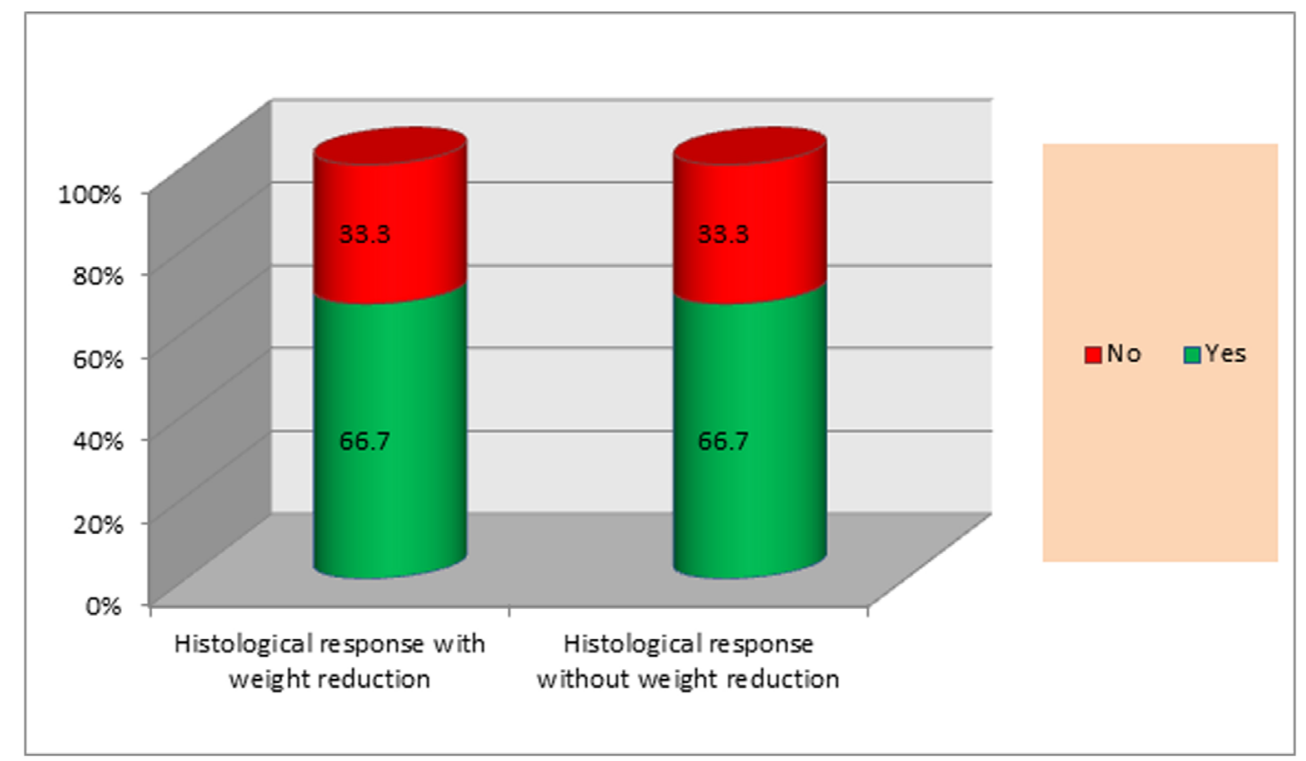

Figure 3: Relation between histological response and $\geq 7 \%$ weight loss

\begin{tabular}{lllll}
\hline Table 4: Predictors of patient response & & \\
\hline & \multicolumn{2}{l}{ Univariate Analysis } & & \multicolumn{2}{c}{ Multivariate Analysis } \\
\cline { 2 - 5 } & $\boldsymbol{P}$ value & OR (95\% CI) & $\boldsymbol{P}$ value & OR (95\% CI) \\
\hline Category of patient (treatment) & 0.012 & $9.33(1.63-53.20)$ & 0.027 & $22.76(1.43-362.40)$ \\
BMI improvement & 0.561 & $1.14(0.73-1.77)$ & 0.281 & $0.67(0.33-1.38)$ \\
HOMA-2 IR improvement & 0.478 & $0.85(0.545-1.33)$ & 0.695 & $1.12(0.644-1.94)$ \\
\hline
\end{tabular}

Abbreviation: BMI: body mass index; HOMA-2 IR: homeostatic model of assessment of insulin resistance.

\begin{tabular}{llll}
\hline Table 5: Safety result & & \\
\hline Side effects & PL group $(\boldsymbol{n}=\mathbf{2 0})$ & L group $(\boldsymbol{n}=\mathbf{1 0})$ & $\boldsymbol{P}$ value \\
\hline Abdominal pain $(\mathrm{Y} / \mathrm{N})$ & $5 / 15$ & $3 / 7$ & 0.086 \\
Dyspepsia(Y/N) & $1 / 19$ & $2 / 8$ & 0.262 \\
\hline
\end{tabular}




\section{DISCUSSION}

This study was performed to observe the effect of pentoxifylline on histological activity of NASH patients and was the $1^{\text {st }}$ Randomized Controlled Trial in the country among NASH patients. Current study prospectively showed that pentoxifylline significantly improved the histology of NASH patients as compared to control group. Our experimental (PL) group received pentoxifylline $1200 \mathrm{mg} /$ day, which was safe and well tolerated as the previous study done by Wegner et al. ${ }^{[21]}$

The severity of steatosis, hepatocellular ballooning and lobular inflammation were quantified by the activity score for NAFLD. ${ }^{[2]}$ The severity of these components decrease with the progression of fibrosis to cirrhosis. ${ }^{[23]}$ So, both NAFLD Activity Score (NAS) as a whole and individual components and fibrosis score was taken into consideration as a significant histological improvement in this study. These strengthen the evaluation of the study outcome.

NAS improved in both PL group and L group. NAS $\geq$ 2 improved in 15 patients out of $20(75 \%)$ in PL group and 3 patients out of $10(30 \%)$ in L group. The difference in terms of responders between PL and L group was statistically significant $(P=0.018)$. This finding was consistent with Zein et al. ${ }^{[24]}$ where NAS $\geq 2$ improved in $50 \%$ of patients on PTX where as $15.4 \%$ of those on placebo, but our study showed a higher degree of improvement. NAS was improved with PTX in another report of Georgescu et al. ${ }^{[15]}$ We found improvement of steatosis and lobular inflammation with PL group in this RCT. These are in accordance with the previous studies. ${ }^{[21,24]}$ Ballooning was not improved in this study and this is similar to those 2 studies. ${ }^{[21,24]}$ The fibrosis score did not improve in PL group and L group. In PL group, fibrosis score $\geq 1$ improved in 4 patients $(20 \%)$, whereas in $\mathrm{L}$ group, it improved in 2 patients $(20 \%)$. This finding was similar with the previous report, ${ }^{[24]}$ where the fibrosis score improvement was not statistically significant.

A large RCT showed that Vitamin E had a significant role in histological improvement of NASH patient. ${ }^{[25]}$ This RCT revealed that Vitamin E improved NAS $\geq 2$ in $43 \%$ of patients. Another 2 RCTs explored that Telmisartan seemed to be efficient in NASH. ${ }^{[26,27]}$ Our RCT revealed that pentoxifylline improved NAS $\geq 2$ in $75 \%$ of patients. So, regarding the improvement of NAFLD Activity Score (NAS), pentoxifylline is more efficacious than Telmisartan and Vitamin E. Fibrosis was also improved with Telmisartan in the previous study. ${ }^{[2]}$ So, regarding the improvement of fibrosis, Telmisartan is superior to pentoxifylline. As PTX is known to decrease oxidative stress, ${ }^{[10]}$ have hydroxyl and peroxyl radical scavenging effects ${ }^{[1]}$ and specifically inhibit lipid peroxidation. ${ }^{[12]}$ PTX blocks the second hit that includes oxidative stress that leads to the secretion of proinflammatory cytokines such as TNF- $\alpha$ and adiponectin, an anti-inflammatory cytokine. They are believed to play an important role in hepatocellular damage, inflammation and fibrogenesis in NASH. These mechanisms of action of PTX are the best explanations of improvement of NAS in our RCT.

Musso et al. ${ }^{[28]}$ described in his meta-analysis research that a significant histological improvement of NASH patient was associated with body weight reduction through sedentary life style changes. But meta-analysis could not quantify the cut off value. Weight reduction of more than $7 \%$ sustained over 48 weeks is associated with significant reduction in histological severity of NASH. ${ }^{[29]}$ As life style modification is the standard approach of patient management, current study included this approach in both groups.

In our study, $7 \%$ or more body weight reduced in 9 out of 30 patients. Weight loss of $7 \%$ or more did not affect the patient's response significantly $(P=0.592)$. Steatosis was improved with this amount of weight loss but it was similar with weight looser and weight non-looser of the abovementioned amount. These findings were not consistent with Lee $e t a l .{ }^{[30]}$ and Wagner $e t$ al. ${ }^{[21]}$ where the weight loss correlated with histological improvement. The underlying cause was not clear, but these findings further strengthen our study that the histological improvements of PTX were not associated with significant weight reduction.

In this RCT, BMI, ALT, AST and GGT was significantly improved after 1 year of intervention with PTX and life style change. The changes of ALT, AST and GGT was independent of weight reduction. Reduction of BMI was previously reported in 2 studies with PTX. ${ }^{[15,30]}$ Reduction ALT and AST with PTX was also in accordance with several previous studies. ${ }^{[24,26,30,31]}$ But reduction of GGT is exceptional in this study that was dissimilar to those studies. Our study could not find any significant effect of PTX on serum cholesterol and triglyceride with 1 year therapy. This was justified with other studies. ${ }^{[24,30,31]}$

In the current study, other bio-chemical parameter such as FBS, 2HABF, HOMA 2-IR, ALT, GGT, Cholesterol, TG, HDL and LDL improvement did not differ significantly among histological responders and non-responders. These findings revealed that bio-chemical improvement does not correlate with histological improvement. All the components of fasting lipid profile improved more in responders than non-responders, but none of them reached up to statistically significant levels. Serum ALT improved both in responders and non-responders. On the contrary, serum GGT improved $24.06 \pm 38.14 \mathrm{U} / \mathrm{L}$ in responders 
and deteriorated $-0.45 \pm 48.74 \mathrm{U} / \mathrm{L}$ in non-responders, but not statistically significant $(P=0.148)$. These findings were not consistent with the finding of Zein et al. ${ }^{[24]}$ and Sanyal et al. ${ }^{[25]}$. In these 2 RCTs, the histological improvements were consistent with the serum ALT improvements. But previous reports from Bangladesh expressed that ALT and AST do not correlate with NAS. ${ }^{[9,32]}$

Regarding safety profile, it revealed that PTX had minimum side effects and similar with $\mathrm{L}$ group. None required treatment discontinuation due to side effects. This finding was consistent with Wagner et al., ${ }^{[30]}$ where adverse events were mild and most frequently abdominal cramp, and were similar in both groups.

This small scale RCT revealed that PTX improved histology significantly; steatosis and lobular inflammation in NASH patient. The main limitation of this study was that it was a small scale open label RCT. All patients were collected from a single tertiary level hospital. So, the current study suffered from lack of multi-centric different ethnic category of patients.

We recommend carrying out large multi-centric double blind RCT to consolidate the findings of this study. In conclusion, this study demonstrates that PTX safely and effectively improves the overall histology, that is, the NAFLD activity score of NASH patient.

\section{Conflict of Interest}

None declared.

\section{REFERENCES}

1. Angulo P. GI epidemiology: nonalcoholic fatty liver disease. Aliment Pharmacol Ther 2007; 25:883-9.

2. Younossi ZM, Koenig AB, Abdelatif D, Fazel Y, Henry L, Wymer M. Global epidemiology of nonalcoholic fatty liver disease - Meta-analytic assessment of prevalence, incidence, and outcomes. Hepatology 2016; 64:73-84.

3. Chan WK, Tan AT, Vethakkan SR, Tah PC, Vijayananthan A, Goh KL. Low physical activity and energy dense Malaysian foods are associated with non-alcoholic fatty liver disease in centrally obese but not in noncentrally obese patients with diabetes mellitus. Asia Pac J Clin Nutr 2015;24:289-98.

4. Alazawi W, Mathur R, Abeysekera K, et al. Ethnicity and the diagnosis gap in liver disease: a population-based study. $\mathrm{Br} \mathrm{J} \mathrm{Gen} \mathrm{Pract} \mathrm{2014;}$ 64:e694-702.

5. Haque MI. NAFLD in Bangladesh. Abstract Book 1st Conference of SASL 2013:69.

6. Rahman MM, Kibria GM, Begum H, et al. Prevalence and Risk Factors of Nonalcoholic Fatty Liver Disease in a Rural Community of South Asia. Gastroenterology 2015;148:S.

7. Adams LA, Lymp JF, St Sauver J, et al. The natural history of nonalcoholic fatty liver disease: a population-based cohort study. Gastroenterology. 2005;129:113-21.
8. Grattagliano I, Portincasa P, Palmieri VO, Palasciano G. Managing nonalcoholic fatty liver disease: recommendations for family physicians. Can Fam Physician 2007; 53: 857-63.

9. Alam S, Noor EASM, Chowdhury ZR, Alam M, Kabir J. Nonalcoholic $\underline{\text { steatohepatitis in nonalcoholic fatty liver disease patients of Bangladesh. }}$ World J Hepatol 2013; 5:281-7.

10. Koppe SW, Sahai A, Malladi P, Whitington PF, Green RM. Pentoxifylline attenuates steatohepatitis induced by the methionine choline deficient diet. J Hepatol 2004;41:592-8.

11. Bhat VB, Madyastha KM. Antioxidant and radical scavenging properties of 8-oxo derivatives of xanthine drugs pentoxifylline and lisofylline. Biochem Biophys Res Commun 2001;288:1212-7.

12. Radfar M, Larijani B, Hadjibabaie M, Rajabipour B, Mojtahedi A, Abdollahi M. Effects of pentoxifylline on oxidative stress and levels of EGF and NO in blood of diabetic type-2 patients; a randomized, double-blind placebo-controlled clinical trial. Biomed. Pharmacother. 2005;59:302-6.

13. Strieter RM, Remick DG, Ward PA, Spengler RN, Lynch III JP, Larrick $\mathrm{J}$, et al. Cellular and molecular regulation of tumor necrosis factor-alpha production by pentoxifylline. Biochem Biophys Res Commun 1988; 155:1230-6.

14. Satapathy SK, Sakhuja P, Malhotra V, Sharma BC, Sarin SK. Beneficial effects of pentoxifylline on hepatic steatosis, fibrosis and necroinflammation in patients with non-alcoholic steatohepatitis. J Gastroenterol Hepatol 2007;22:634-8.

15. Georgescu EF, Georgescu M. Therapeutic options in nonalcoholic steatohepatitis (NASH). Are all agents alike? Results of a preliminary study. J Gastrointestin Liver Dis 2007; 16: 39-46.

16. Du J, Ma YY, Yu CH, Li YM. Effects of pentoxifylline on nonalcoholic fatty liver disease: a meta-analysis. World J Gastroenterol 2014;20:569-77.

17. Matthews DR, Hosker JP, Rudenski AS, Naylor BA, Treacher DF, Turner RC. Homeostasis model assessment: insulin resistance and beta-cell function from fasting plasma glucose and insulin concentrations in man. Diabetologia 1985;28:412-9.

18. Kleiner DE, Brunt EM, Van Natta M, et al. Design and validation of a histological scoring system for nonalcoholic fatty liver disease. Hepatology 2005;41:1313-21.

19. Grant A, Neuberger J. Guidelines on the use of liver biopsy in clinical practice. British Society of Gastroenterology. Gut 1999;45 Suppl 4:Iv1-iv11.

20. Mustafa G, Alam S, Al Mamun A, Ahmad N, Alam K, Khan M. Percutaneous liver biopsy: technique and safety. Hepatogastroenterology 2011;58:529-31.

21. Van Wagner LB, Koppe S, Brunt EM, Gottstein J, Gardikiotes K, Green $\mathrm{RM}$, et al. Pentoxifylline for the treatment of non-alcoholic steatohepatitis: a randomized controlled trial. Ann Hepatol 2011; 10: $277-$ 286.

22. Adams LA, Talwalkar JA. Diagnostic evaluation of nonalcoholic fatty liver disease. J Clin Gastroenterol 2006;40Suppl 1:S34-8.

23. Bacon BR, Farahvash MJ, Janney CG, Neuschwander-Tetri BA. Nonalcoholic steatohepatitis: an expanded clinical entity. Gastroenterology. 1994;107:1103-9.

24. Zein CO, Yerian LM, Gogate P, Lopez R, Kirwan JP, Feldstein AE, et al. Pentoxifylline improves nonalcoholic steatohepatitis: a randomized placebo-controlled trial. Hepatology 2011;54:1610-9.

25. Sanyal AJ, Chalasani N, Kowdley KV, McCullough A, Diehl AM, Bass NM, et al. Pioglitazone, vitamin E, or placebo for nonalcoholic steatohepatitis. N England J Med 2010;362:1675-85.

26. Georgescu EF, Ionescu R, Niculescu M, Mogoanta L, Vancica L. Angiotensin-receptor blockers as therapy for mild-to-moderate hypertension-associated non-alcoholic steatohepatitis. World J Gastroenterol 2009;15:942-54.

27. Alam S, Kabir J, Gupta UD, Mustafa G, Hasan SKM, Alam AKM. Effect of Telmisartan on Histological Activity and Fibrosis of Nonalcoholic Steatohepatitis Patients - A 1Year Randomized Control Trial. Saudi J Gastroenterol 2016; 22:69-76. 
28. Musso G, Gambino R, Cassader M, Pagano G. A meta-analysis of randomized trials for the treatment of nonalcoholic fatty liver disease. Hepatology 2010;52:79-104.

29. Promrat K, Kleiner DE, Niemeier HM, Jackvony E, Kearns M, Wands $\mathrm{JR}$, et al. Randomized controlled trial testing the effects of weight loss on nonalcoholic steatohepatitis. Hepatology 2010;51:121-9.

30. Lee YM, Sutedja DS, Wai CT, Dan YY, Aung MO, Zhou L, et al. A randomized controlled pilot study of Pentoxifylline in patients with non-alcoholic steatohepatitis (NASH). Hepatol Int 2008;2:196-201.
31. Tuncer İ, Uygan İ, Dülger H, Türkdo ğ an K, Şekero ğ lu MR, Kösem M The comparative effects of pentoxifylline and ursodeoxycholic acid on IL-1 $\beta$, Il-6, Il-8 and TNF- $\alpha$ levels in nonalcoholic fatty liver. E J Med 2003;8:27-32.

32. Alam S, Gupta UD, Kabir J, Alam SMNE, Chowdhury ZR, Alam AKMK. Transaminases and Gamma Glutamyl Transpeptidase for Detecting Nonalcoholic Steatohepatitis and Fibrosis in Nonalcoholic Fatty Liver Disease. BSMMU J 2015; 8: 61-7.

How to cite this article: Alam S, Hasan SKMN, Mustafa G, Alam M, Kamal M, Ahmad N. Pentoxifylline in Nonalcoholic Steatohepatitis. J Transl Intern Med 2017; 5: 155-163. 